\title{
Galaxy shape measurement with convolutional neural networks
}

\author{
Dezső Ribli ${ }^{1 \star}$, László Dobos ${ }^{1}$ and István Csabai ${ }^{1}$ \\ ${ }^{1}$ Eötvös Loránd University, Institute of Physics, Budapest, Hungary
}

Accepted XXX. Received YYY; in original form ZZZ

\section{INTRODUCTION}

Light from distant galaxies is deflected by the tidal fields of inhomogeneous matter along the line of sight, distorting the shapes of sources, a phenomenon called gravitation lensing. Cosmic shear is the weak gravitational lensing effect arising from no obvious foreground mass apart from the large scale structure of the Universe. Detecting the effects of the foreground mass density field on distant galaxy images allows us to indirectly map the distribution of the elusive, and apparently very abundant dark matter. Furthermore, characterising matter distribution at different redshifts through the lensing signal offers a unique window to the evolution of the dark energy dominated late Universe, complementary to other observations (see Mandelbaum et al. (2018); Kilbinger (2015) for recent reviews).

When the lensing effect of foreground matter is strong

^ E-mail: dkrib@caesar.elte.hu (DR) enough, it can dramatically alter the appearance of background sources, making the lensing signal detectable even for individual galaxies. The large scale structure, however, only distorts the shapes of background objects with a small, approximately linear shear, rendering the lensing signal indistinguishable from intrinsic shape variations of lensed galaxies. The spatial correlations in the apparent shapes of galaxies introduced by the large scale structure make cosmic shear statistically measurable from an ensemble of galaxies. Since the first detection of cosmic shear about two decades ago (Bacon et al. 2000; Kaiser et al. 2000; Van Waerbeke et al. 2000; Wittman et al. 2000) weak lensing measurements have matured into a potent probe of cosmology. Weak lensing survey volumes increased constantly, with the COSMOS field and Hubble Space Telescope images (Schrabback et al. 2010) followed by CFHTLenS ${ }^{1}$ which was the first major weak 
lensing survey pushing the number of galaxies with highquality shape measurements to the millions by covering an area of 154 square degrees at a resolved galaxy density of 17 per square arc minute. Shapes of hundreds of millions of galaxies are being measured by ongoing weak lensing surveys, such as the Dark Energy Survey (DES) ${ }^{2}$, the KiloDegree Survey (KiDS) ${ }^{3}$, and the Subaru Hyper SuprimeCam Survey (HSC) ${ }^{4}$, whereas billions more will be observed by large surveys that are under construction, such as the Large Synoptic Survey Telescope (LSST) ${ }^{5}$, or in the development phase, such as the Euclid mission ${ }^{6}$ or the Wild Field Infrared Survey (WFIRST) ${ }^{7}$.

Cosmic shear only distorts the shapes of galaxies at percent levels (Kilbinger 2015), and the signal is dominated by noise from intrinsic shape variation of galaxies, atmospheric and instrumental distortions. The bulk of the cosmic shear signal is carried by shapes of small and faint galaxies typically those with sizes of a few arcsecs and 24 magnitudes (Kilbinger 2015). Weak lensing surveys, therefore, need to accurately estimate the shapes of galaxies which only cover a few pixels and have very low signal to noise ratios. Shape measurements are further complicated by the smearing of the original galaxy images by atmospheric and instrumental point spread functions (PSF). The PSF itself has a coherent spatially and temporally variable anisotropy mimicking the effect of cosmic shear, therefore PSF anisotropy must be carefully measured and corrected for precise shear estimation (Miller et al. 2013).

Popular shape measurement algorithms fall in two main categories, the first approach attempts to directly measure the shapes of galaxies through second-order moments of the surface brightness profile (Kaiser et al. 1995). Another approach, dominantly used in recent weak lensing surveys, uses forward model fitting, where parametric surface brightness model profiles are convolved with the PSF and compared to measurements (Kuijken 1999; Miller et al. 2007; Kitching et al. 2008; Miller et al. 2013; Kuijken et al. 2015; Hildebrandt et al. 2017; Fenech Conti et al. 2017; Zuntz et al. 2018). Model fitting is preferred over measuring moments due to the efficient handling of the PSF and the joint fit over multiple exposures (Heymans et al. 2012) or colours (Zuntz et al. 2018). In model fitting approaches it is convenient to parametrise the shapes of galaxies with the shear style complex ellipticity $\boldsymbol{\epsilon}$, which is an unbiased estimator of weak shear.

$\epsilon_{1}+i \epsilon_{2}=\frac{a-b}{a+b} e^{i 2 \theta}$

, where $a$ is the length of the semi-major axis, $b$ is the length the semi-minor axis and $\theta$ is the position angle of an ellipse.

The redshifts of galaxies also need to be measured for optimal extraction of the cosmological signal, however, this is not possible with spectroscopy due to the vast number of

\footnotetext{
2 https://www.darkenergysurvey.org

3 http://kids.strw.leidenuniv.nl

4 https://hsc.mtk.nao.ac.jp/ssp/

5 http://www.lsst.org/

6 http://sci.esa.int/euclid/

7 http://wfirst.gsfc.nasa.gov/
}

galaxies used, and weak lensing surveys need to rely on photometric redshift estimates from multi-band observations. Shapes of galaxies are highly correlated in different colors and information from multiple bands can be combined to improve shape measurements (Jarvis \& Jain 2008). The feasibility of this approach was demonstrated in one of the DES Y1 shape catalogues (Zuntz et al. 2018).

Pixel values of a galaxy image have complex non-linear relationship with ellipticity parameters and the likelihood surface is skewed towards zero ellipticity and towards the ellipticity of the PSF. This effect introduces a bias in the maximum likelihood estimate or the expected value of the shape parameters, which becomes significant for noisy galaxies, hence it is called the "noise bias" (Bernstein \& Jarvis 2002; Kitching et al. 2010a; Melchior \& Viola 2012; Refregier et al. 2012; Kacprzak et al. 2012; Miller et al. 2013). Following Heymans et al. (2006), bias in ensemble shear measurements is usually parametrized with a linear function.

$\left\langle\epsilon_{i}^{o b s}\right\rangle=(1+m) g_{i}+c+\alpha \epsilon_{i}^{P S F}$

, $i$ denotes the component of the ellipticity $(1,2), g$ is the true cosmic shear, the intercept $c$ is called the additive bias, the excess slope $m$ is the multiplicative bias and $\alpha$ is the PSF leakage. The additive bias and the PSF leakage are strongly related and they are corrected using measurement data (Heymans et al. 2012), however, the multiplicative term is generally mitigated with simulations.

The multiplicative bias strongly depends on the observed $\mathrm{S} / \mathrm{N}$ and size of galaxies and the precise relationship is identified through estimating the properties of millions of galaxy images simulated with sophisticated tools (Rowe et al. 2014) and known shape parameters (Miller et al. 2013; Jarvis et al. 2016; Fenech Conti et al. 2017; Zuntz et al. 2018; Pujol et al. 2019). These simulations need to be specifically tailored to the details of surveys, and the calibration process has to be conducted for every single survey or data release. After the precise nature of the bias is established, empirical corrections of measured galaxy ellipticities are applied to negate the bias.

The DES Y1 shape catalogue (Zuntz et al. 2018) employed an innovative approach called METACALIBRATION, which only uses observational data and post measurement shear operations and convolutions to correct for noise bias (Huff \& Mandelbaum 2017; Sheldon \& Huff 2017). METACALIBRATION offers a principled shear calibration framework without large numbers of calibration factors derived from simulations, with sufficiently small multiplicative bias even for large future weak lensing surveys (Sheldon \& Huff 2017). The PSF anisotropies are handled during the deconvolution step in METACALIBRATION, which allows the calibration of any well-behaving estimator (Sheldon \& Huff 2017), such as adaptive moments (Bernstein \& Jarvis 2002), not only model fitting with forward convolutions.

Apart from noise bias, selection biases also appear when galaxies are preferentially detected depending on the alignment of their shapes, the anisotropy of the PSF and lensing shear (Kaiser 2000; Hirata \& Seljak 2003). Selection bias may also affect detected galaxies due to the fact that the ellipticity likelihood surface is narrower for galaxies if the intrinsic and PSF ellipticities are aligned. Methods which assign an inverse-variance weight to shape measurements 
based on the width of the likelihood surface (Miller et al. 2013; Fenech Conti et al. 2017), favour galaxies parallel to the PSF, creating a selection bias in the shear.

Shape measurement approaches which assume simplified galaxy surface brightness profiles potentially suffer from 'model bias' if they are not able to capture the shapes of complex galaxies (Voigt \& Bridle 2010; Melchior et al. 2010). Model bias is expected to have only a minor contribution in ground-based observations (Miller et al. 2013; Mandelbaum et al. 2015), and it was explored in detail for model fitting shape measurements (Zuntz et al. 2013; Kacprzak et al. 2014).

Biases in the shape measurements could systematically alter cosmological parameters inferred from weak lensing measurements and the community understandably paid particular attention to the question of systematic biases, also in the form of organized challenges (Heymans et al. 2006; Massey et al. 2007; Bridle et al. 2009, 2010; Kitching et al. 2010b,a; Simet et al. 2014; Mandelbaum et al. 2015). However, shape measurements not only need to be unbiased but also they need to have small variance in order to reduce statistical uncertainty, a quantity often expressed in terms of an effective galaxy surface density (Heymans et al. 2012; Chang et al. 2013). Large efforts are ongoing and planned (HSC, LSST, Euclid, WFIRST) to conduct very deep surveys which aim to radically increase the galaxy surface density and the statistical power of measurements (Takada 2010; Chang et al. 2013; Amendola et al. 2018). Increased galaxy densities enable more precise measurements of cosmic shear at high angular resolutions, allowing the extraction of significant non-Gaussian information, which can further empower weak lensing measurements (Ribli et al. 2019a).

The variance of shape measurements received much less attention than their bias, probably because one might think that model fitting with forward convolution is an optimal method. However, noise in galaxy shape measurements is known to be not a completely independent shot noise (Mandelbaum et al. 2015) and model fitting approaches can only work with simplified parametric surface brightness profiles (Miller et al. 2013; Zuntz et al. 2018), which may be suboptimal for faint irregular galaxies, abundant in weak lensing surveys. Systematic biases in current surveys are significantly smaller than statistical uncertainties (Mandelbaum et al. 2015; Zuntz et al. 2018; Fenech Conti et al. 2017), therefore shape measurements with smaller dispersion could further reduce the uncertainty of underlying cosmological parameters.

Maximum likelihood model fitting with forward convolutions takes approximately 1.6 seconds per galaxy per image (Zuntz et al. 2013), consuming 200,000 CPU hours for the DES Y1 IM3shape shape catalogue. The final DES survey is expected to include an order of magnitude more galaxies, and near future surveys such as LSST and Euclid will again grow an order of magnitude over that number. Including multiple colors in the fitting process or handling approximately 1000 individual observations per galaxy in LSST further boost the computational burden of maximum likelihood model fitting. In order to handle the large amount of data expected in future surveys, extremely fast shape estimators are needed which can handle multi-epoch data efficiently.

This work focuses on reducing the statistical dispersion of shape estimates, via convolutional neural networks (CNN) which recently revolutionized the field of computer vision and reached human level accuracy in image classification (He et al. 2015). CNNs are sophisticated machine learning methods which are able to learn from a large number of labelled images, and apart from image classification they also excel in image deconvolution or "super-resolution" in the presence of noise for everyday images (Xu et al. 2014) or microscopy (Wang et al. 2019). We construct a large labelled dataset using images from a wide survey, DES DR1 (Abbott et al. 2018; Morganson et al. 2018), and high quality galaxy shape measurements from a significantly deeper survey with overlapping footprint, CFHTLenS (Erben et al. 2013; Heymans et al. 2013; Hildebrandt et al. 2012; Miller et al. 2013). The $\mathrm{CNN}$ is trained to predict ellipticities of galaxies measured by the deeper survey using images of the wide survey as inputs.

CNNs evaluate an image with a single forward pass though their layers, while making use of massively parallel computer hardware (GPU, TPU) which allow submillisecond execution times per galaxy, orders of magnitude faster than model-fitting approaches. The extreme speed of CNN based estimation alone warrants the exploration of this approach, as it may be able to handle the expected data surge and exploit all the information available at the same time in near future large weak lensing surveys.

Multiple studies explored recently machine learning and CNNs in problems related to weak gravitational lensing or the estimation of galaxy properties Dieleman et al. (2015) used custom rotation invariant CNNs for galaxy morphology prediction using ground truth values determined by citizen scientist in the Galaxy Zoo project (Lintott et al. 2008). CNNs were used to infer the morphological parameter of simulated galaxies (Tuccillo et al. 2017), with an improved catalogue released for SDSS (Domínguez Sánchez et al. 2018). Herbel et al. (2018) use CNNs for the estimation of PSF shape parameters to enable fast PSF modelling. Springer et al. (2018) train CNNs to directly predict relatively strong simulated shears in large, resolved galaxy images in the context of galaxy cluster lensing. Most recently Tewes et al. (2019) train neural networks to calibrate shear measurements using biased shape estimators such as second order moments and various galaxy parameters as inputs. The approach used by Tewes et al. (2019) is complementary to our work and it could use the output of a shape estimator CNN to achieve potentially more accurate shear estimators with small bias.

The outline of the paper is the following: in $\S 2$ we describe the dataset used for training and testing, the details of the CNN architecture and the training process. In $\S 3$ we train a shape estimator CNN on single band images and compare it to the DES Y1 IM3SHAPE catalogue depending of various measured factors. In $\S 4$ we train the $\mathrm{CNN}$ on multiple band images and compare it to the DES Y1 METACALIBRATION catalogue. In $\S 5$ we determine the necessary training dataset size. In $\S 6$ we test the biases of the CNN shape estimator nested in METACALIBRATION using a large suit of simulated galaxies, and finally we discuss the results in $\S 7$. 

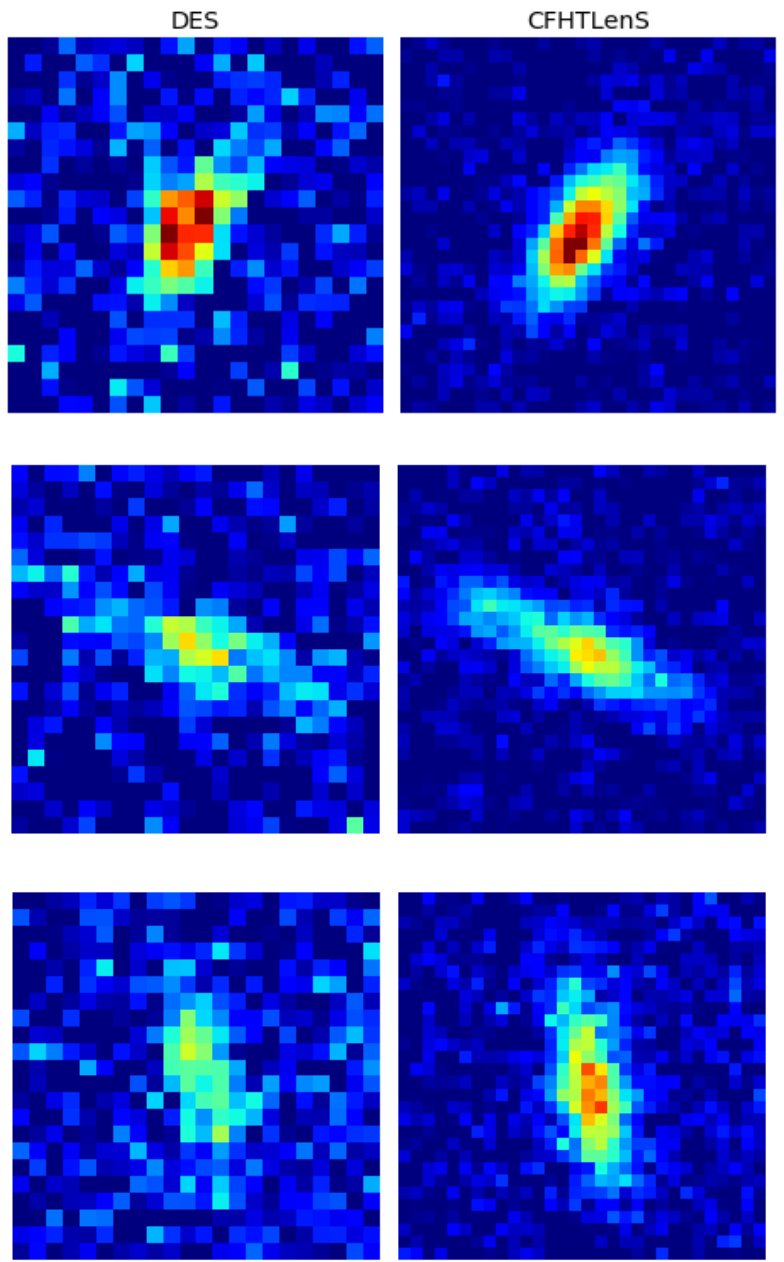

Figure 1. Example galaxies on DES (left) and the deeper CFHTLenS survey(right) images in the $i$-band. CFHTLenS images have higher $\mathrm{S} / \mathrm{N}$ and they allow more accurate shape measurements of the same galaxies. The galaxies have $i$-band magnitudes $(22.05,22.09,22.18)$, top to bottom.

\section{DATA AND METHODS}

\subsection{Observational data}

We select galaxies from DES Y1 shape catalogues which are also in the overlapping W4 region of CFHTLenS. The DES Y1 shape catalogues (IM3Shape,MetaCALIBRATION) (Zuntz et al. 2018), are cross matched with the CFHTLenS shape catalogue (Erben et al. 2013) using Skyquery, a probabilistic join engine for cross-identification of multiple astronomical databases (Dobos et al. 2012). We only use galaxies with confident CFHTLenS shape measurements, specifically, where the inverse variance weight is larger than 14 . The final dataset contains $\approx 1.4 \times 10^{5}$ galaxies.

The corresponding DES DR1 stacked multi epoch images (Abbott et al. 2018) were downloaded for each galaxy and 48 pixel sized postage stamps were cut out in each of the 'grizY' DES bands. Stacked multi epoch images are considered sub-optimal for shear measurement and were recently replaced by joint fitting of multiple single exposures for the analysis of weak lensing surveys (Heymans et al. 2012; Miller

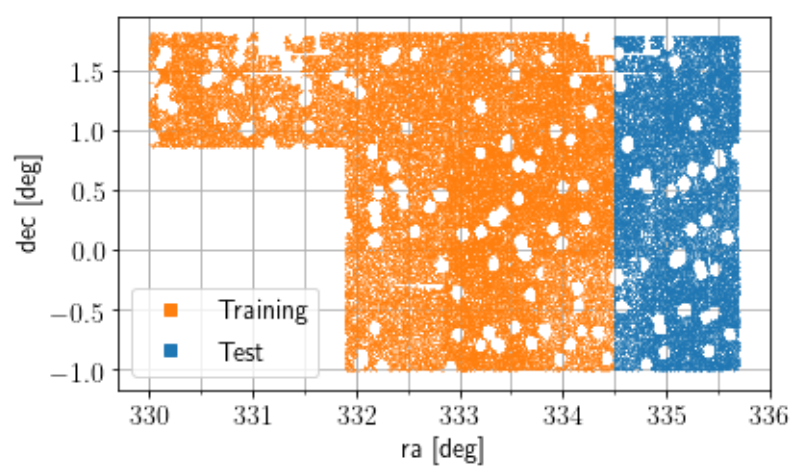

Figure 2. We use non-overlapping training and test regions to evaluate generalisation to new regions in the sky.

et al. 2013; Kuijken et al. 2015; Fenech Conti et al. 2017; Hildebrandt et al. 2017; Zuntz et al. 2018), however, these images are suitable for evaluation of the dispersion of shape estimates. Potential biases associated with dithered stacked images due to PSF discontinuities and interpolation (Miller et al. 2013) are not important here as we do not attempt to go further than shapes of individual galaxies, and we do not calculate ensemble shear estimates. The depth of the Y1 and the DR1 releases of DES are similar in the part of the equatorial region used in this study, as Y2 and Y3 operations concentrated on other regions of the sky (Diehl et al. 2016).

We define highly confident ellipticity parameters measured with lensfit using the CFHTLenS $i$ band images (Miller et al. 2013) as the 'ground truth' labels to train our neural network. The two surveys use telescopes with similar mirror sizes, and CFHTLens $i$ band data was integrated for 4300 seconds, while the DES DR1 data has 450 second integrated exposure in each of the griz bands. The CFHTLenS survey is approximately 1 magnitude deeper than DES DR1 (Erben et al. 2013; Abbott et al. 2018), which allows much more precise shape measurements for faint galaxies. The difference in image quality for faint galaxies is demonstrated visually with examples on [Fig. 1].

We split the dataset into a $70 \%$ training and a $30 \%$ test set based on the position on the sky [Fig. 2], in order to evaluate the capability of the CNN to generalize to a new region, other than the one used for training. Generalization to other regions of the sky is essential in our proposed scheme where the network is trained on dedicated deep fields of surveys but used for estimation on the whole wide field survey.

\subsection{Evaluation}

For calibration purposes, the 'accuracy' of shape measurement algorithms is often characterized by the values of multiplicative and additive bias. However, in this study we set out to reduce the variance, the statistical dispersion of shape measurements, therefore we choose a different metric which 


\begin{tabular}{rlr}
$\#$ & Layers & Output size \\
\hline 1 & Convolution $(3 \times 3)$ & $48 \times 48 \times 16$ \\
2 & Convolution $(3 \times 3)$ & $48 \times 48 \times 16$ \\
3 & Convolution $(3 \times 3)$ & $24 \times 24 \times 32$ \\
4 & Convolution $(3 \times 3)$ & $24 \times 24 \times 32$ \\
5 & Convolution $(3 \times 3)$ & $12 \times 12 \times 64$ \\
6 & Convolution $(1 \times 1)$ & $12 \times 12 \times 32$ \\
7 & Convolution $(3 \times 3)$ & $12 \times 12 \times 64$ \\
8 & Convolution $(3 \times 3)$ & $6 \times 6 \times 128$ \\
9 & Convolution $(1 \times 1)$ & $6 \times 6 \times 64$ \\
10 & Convolution $(3 \times 3)$ & $6 \times 6 \times 128$ \\
11 & Convolution $(3 \times 3)$ & $3 \times 3 \times 256$ \\
12 & Convolution $(1 \times 1)$ & $3 \times 3 \times 128$ \\
13 & Convolution $(3 \times 3)$ & $3 \times 3 \times 256$ \\
- & Average Pooling $(3 \times 3)$ & $1 \times 1 \times 256$ \\
14 & Dense & 2
\end{tabular}

Table 1. Neural network architecture. Number of trainable parameters: 837,586 .

reflects the covariance of ground truth and estimated ellipticities: the Pearson correlation coefficient.

$\rho(x, y)=\frac{\operatorname{cov}(x, y)}{\sigma_{x} \sigma y}$

We do acknowledge the immense importance of bias correction, which must be thoroughly performed for credible shear measurements, and we demonstrate that CNN shape estimates have sufficiently small bias for future large surveys when implemented in the METACALIBRATION framework. We also expect that CNN shape estimates can just as well be calibrated using a large number of realistic image simulations as any other well-behaving shape estimator. Additional parameters for calibration, such as signal to noise ratio and size could also be predicted by the CNN, similarly to shape, or they could be derived from other tools such as SExtractor (Bertin \& Arnouts 1996). For galaxy level comparisons performed in the present study, bias corrections are negligible compared to the intrinsic dispersion of galaxy ellipticities.

\subsection{Convolutional neural network, training and testing}

We design a custom CNN architecture specifically for the task, building on successful image classification CNNs (Krizhevsky et al. 2012; Simonyan \& Zisserman 2014; Szegedy et al. 2015; He et al. 2016; Redmon \& Farhadi 2017). The network consists of subsequent sliding window filter matching operations, called convolutional layers, which can powerfully express the translation invariance of image data. The width of convolutional filters is $3 \times 3$ pixels for the majority of layers, as this filter width makes most effective use of parameters (Simonyan \& Zisserman 2014). Starting from the fifth layer these $3 \times 3$ convolutional layers are preceded by a bottleneck $1 \times 1$ "convolutional" layer, which compresses input representations to half the number of filters to save computation time and reduce the number of overall parameters. These bottleneck layers are found in almost every single modern CNN architecture (Szegedy et al. 2015; He et al. 2016; Redmon \& Farhadi 2017).

Each convolutional layer, except for the last one, is followed by batch normalization (Ioffe \& Szegedy 2015), which rescales activations in the previous layer in order to stabilize and facilitate training. Batch normalizations are followed by non-linear activation functions called Recitified Linear Units (ReLU), which take the form $\max (0, x)$. The flat part creates non-linearity, which allows the neural network to approximate complex non-linear functions, while the linear region provides stable, non-vanishing gradients when propagated through very deep networks (Krizhevsky et al. 2012). After every 2-3 convolutional layers, the representations are spatially downsampled by only evaluating the convolutional filters at every second position. Downsampling helps to aggregate localized lower level information into higher semantic levels with lower spatial resolution. We start with 16 filters in the first layer and double the number of filters after each spatial downsampling, which is a common practice in deep CNNs. We find that doubling or quadrupling the number of filters in every layer slightly improves the accuracy of the network, however, we settle with 16 filters to avoid additional runtime. When the spatial extent of convolutional layers becomes very small $(3 \times 3)$ we average all activations along spatial dimensions, creating a single one-dimensional representation. Finally, a linear layer with 2 outputs predicts the ellipticity components of the galaxy. The outline of the neural network is detailed in [Tab. 1].

The inputs to the neural network are $48 \times 48$ pixel postage stamp images with pixel values rescaled by the means and r.m.s. values of the sky measured with SExtractor (Bertin \& Arnouts 1996). When training with multiband images, different colors are stacked as different input channels similarly to RGB images. We do not incorporate pixel weights or masks into out inputs, however, we note that it would be straightforward to simply stack these as additional channels of the input image. During training, we augment the dataset with random horizontal and vertical flips and random transpositions to combat overfitting, ground truth ellipticities of galaxies are transformed accordingly during augmentation. The augmentation scheme creates an $8 \times$ larger dataset, however, the new examples are not independent. The applied transformations enable the neural network to learn additional symmetries of the dataset alongside translation invariance represented with convolutional operations. An interesting branch of research efforts attempt to create neural networks with built-in representation for rotational symmetries (Cohen \& Welling 2016; Kondor et al. 2018), however, these works have not completely matured yet. During testing, we evaluate each galaxy and its 90 degrees rotated version, which reduces the errors by only approximately $1 \%$. Evaluating a rotated version doubles execution time and results in only a modest improvement in accuracy, however, we prefer to apply this augmentation because it potentially mitigates additive bias due to its symmetry. We do not predict on the other 6 flipped and transposed versions of the image, because we find only very small improvements from additional test time augmentation, and it makes inference significantly slower.

We train the neural network with a minibatch size of 512 for 40 complete iterations on the training dataset, called an epoch. The training dataset is reshuffled before each traversal to create a varying composition of minibatches in each iteration, which has a regularization effect when batch normalizations are used (Ioffe \& Szegedy 2015). We use stochastic gradient descent optimisation with an initial learning rate 

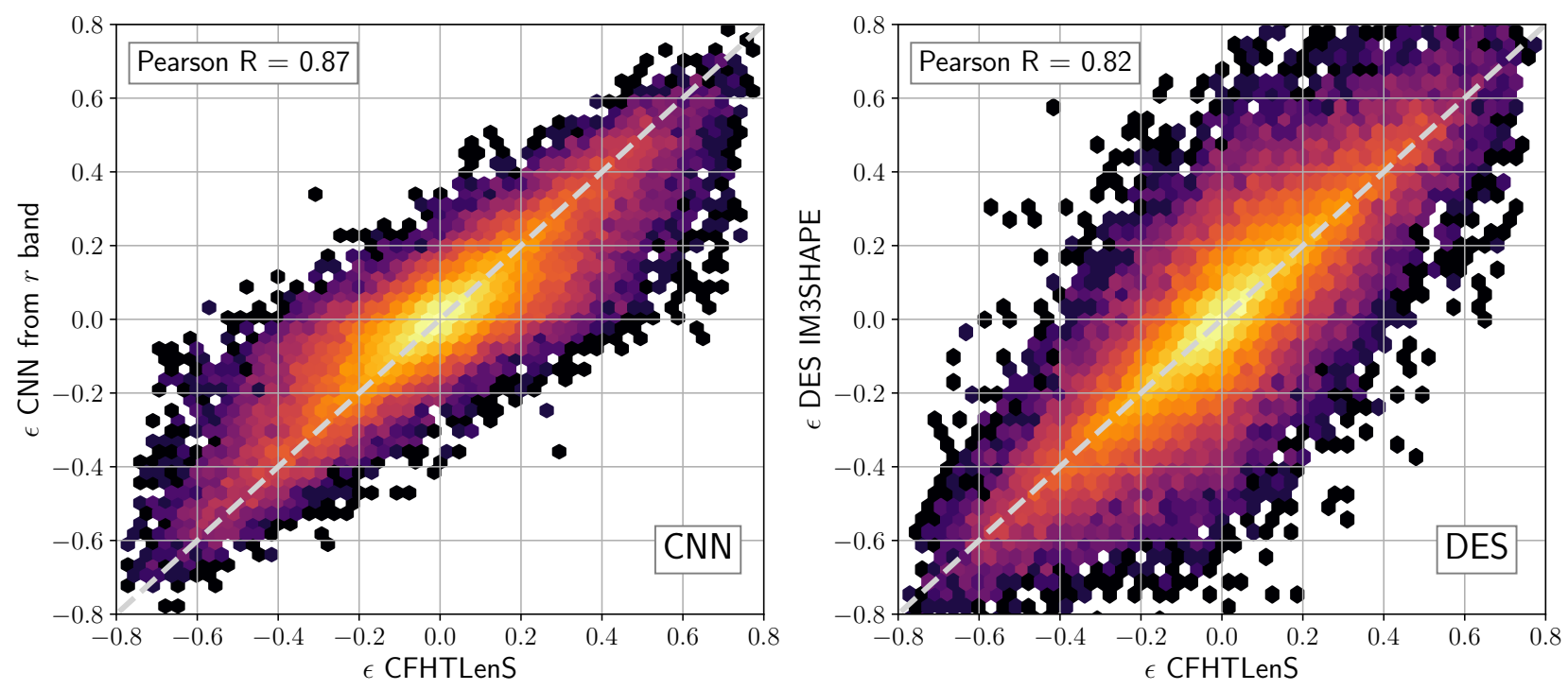

Figure 3. The CNN estimates galaxy shapes with smaller dispersion than the DES IM3SHAPE catalogue. The heatmap shows joint distributions of 'ground truth CFHTLenS shapes and estimates by the CNN (left) and the DES shape catalog (right) for the $40 \%$ of galaxies which were selected for cosmology in the DES IM3SHAPE catalog.

of 0.005 and we decrease the learning rate tenfold after the 20 th and the 30th epoch to enable convergence by the end of the training schedule. During training, we minimise the mean absolute error between predicted and target ellipticities. We do not exhaustively optimize the training process of the neural network by varying hyper-parameters, nor do we attempt to find the optimal CNN architecture. We find that our base setup is very fast and reasonably accurate, and we delegate fine-tuning of the network details to later works.

Our CNN does not use the known PSF during training or predictions. The effect of PSF anisotropy is very small compared to the overall variance of shape estimation, and it absolutely does not effect our results, when comparing the shapes of individual galaxies, without ensembling for shear estimation. Convolutional neural networks are flexible methods and they could be used to produce predictions with low bias and small PSF leakage using special loss functions or similar methods as in Tewes et al. (2019). However we leave this topic to future work, and in this work we demonstrate that PSF anisotropy and multiplicative bias can be handled by nesting our CNN ellipticity estimator within the METCALIBRATION framework, which can be wrapped around any sufficiently stable ellipticity estimator. We find that our results are indistinguishable for the two ellipticity components, therefore our results are shown after concatenating the two components into one ellipticity value. We make source code for our approach publicly available on github ${ }^{8}$.

\section{SHAPE ESTIMATION USING SINGLE BAND IMAGES}

First, we train and evaluate the CNN shape estimator on only $r$ band stacked DES images, enabling fair comparison with the DES IM3SHAPE catalogue. IM3SHAPE is a for-

\footnotetext{
${ }^{8}$ https://github.com/riblidezso/shearNN
}

ward fitting algorithm, where galaxies are modelled with a bulge or a disc profile and profiles are convolved with the PSF before being compared to measured images. The maximum likelihood solutions are found using the LevenbergMarquardt algorithm (Zuntz et al. 2013). For the DES Y1 IM3SHAPE catalogue, shape measurements were performed on multi epoch $r$ band images (Jarvis et al. 2016), and the best fitting bulge or disc models were selected. A large number of galaxies were flagged unfit for cosmology mainly due to their small size and low signal to noise ratio (Jarvis et al. 2016), resulting in unreliable shape estimates, which prohibits accurate bias calibration. The clean sample only includes around $40 \%$ of galaxies detected both in DES and CFHTLens. A large portion of these excluded galaxies are selected for cosmology in the deeper METACALIBRATION catalogue. Heatmaps of true and estimated values show that the predictions of the CNN show smaller dispersion than the DES IM3SHAPE catalogue [Fig. 3] for the high-quality sample selected for cosmology.

In order to understand the performance of the CNN shape estimator under various circumstances, we first investigate the accuracy of shape estimates at different magnitudes. We group galaxies into 10 equal size bins, assign their mean $r$ magnitude to the bin and we calculate the Pearson correlation of estimated and ground truth shapes in each bin [Fig. ] 4]. Note, that for these tests we make predictions with the same model, trained on the whole training set, and we only divide galaxies into different bins for the evaluation. We find that the DES catalogue is just as accurate as the CNN for bright galaxies, and the CNN becomes significantly more precise as noise begins to dominate images. For clarity, we evaluate the above-mentioned figure for three sets, the full catalogue, the subset selected for cosmology and the one excluded. Reassuringly, we find that the subset selected for cosmology is much more accurate than the excluded one, and IM3SHAPE even outperforms the CNN on the brightest galaxies, and only falls behind for much more abundant faint 

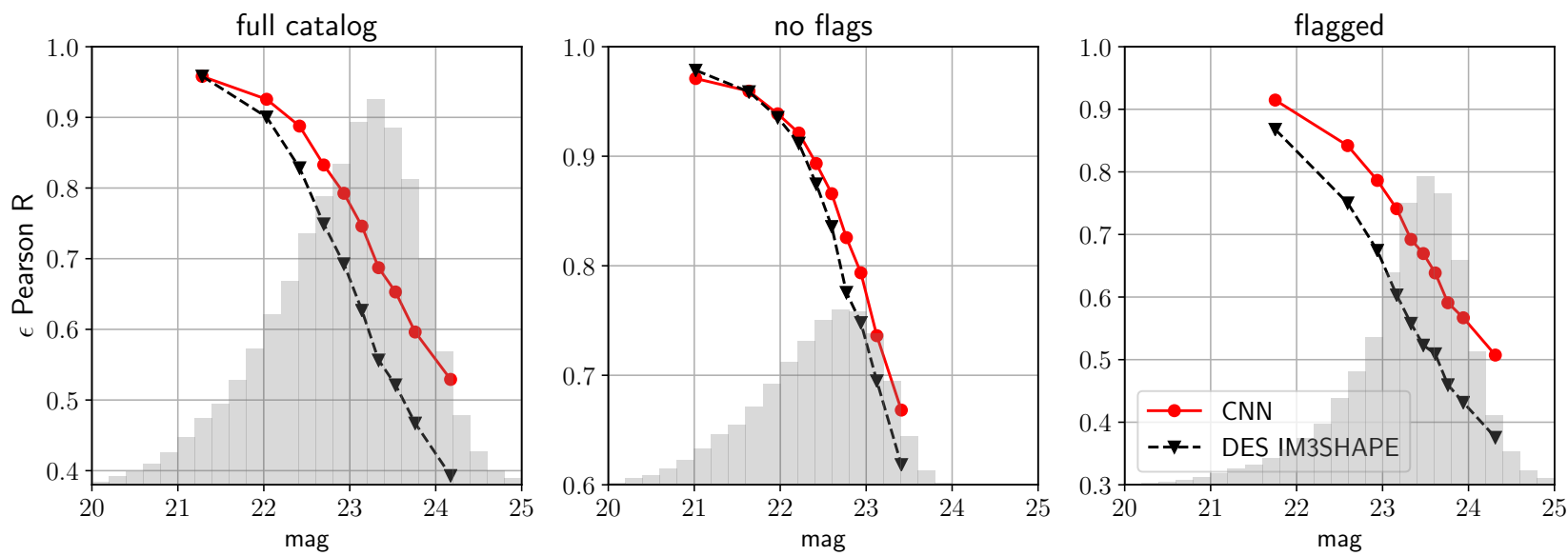

Figure 4. The shapes of faint galaxies are estimated more accurately by the CNN than the DES IM3SHAPE catalog. Pearson correlation between ground truth CFHTLenS and estimated ellipticities are shown depending on the $r$ band magnitude. The histogram of galaxies are shown in light grey. On the $40 \%$ subset selected for cosmology, IM3SHAPE gives slightly more accurate estimates for bright galaxies, but the CNN is more accurate on much more common faint galaxies (center). On the subset excluded from cosmology, mainly due to small size and low brightness (right), the CNN significantly outperforms the DES IM3SHAPE catalogue.
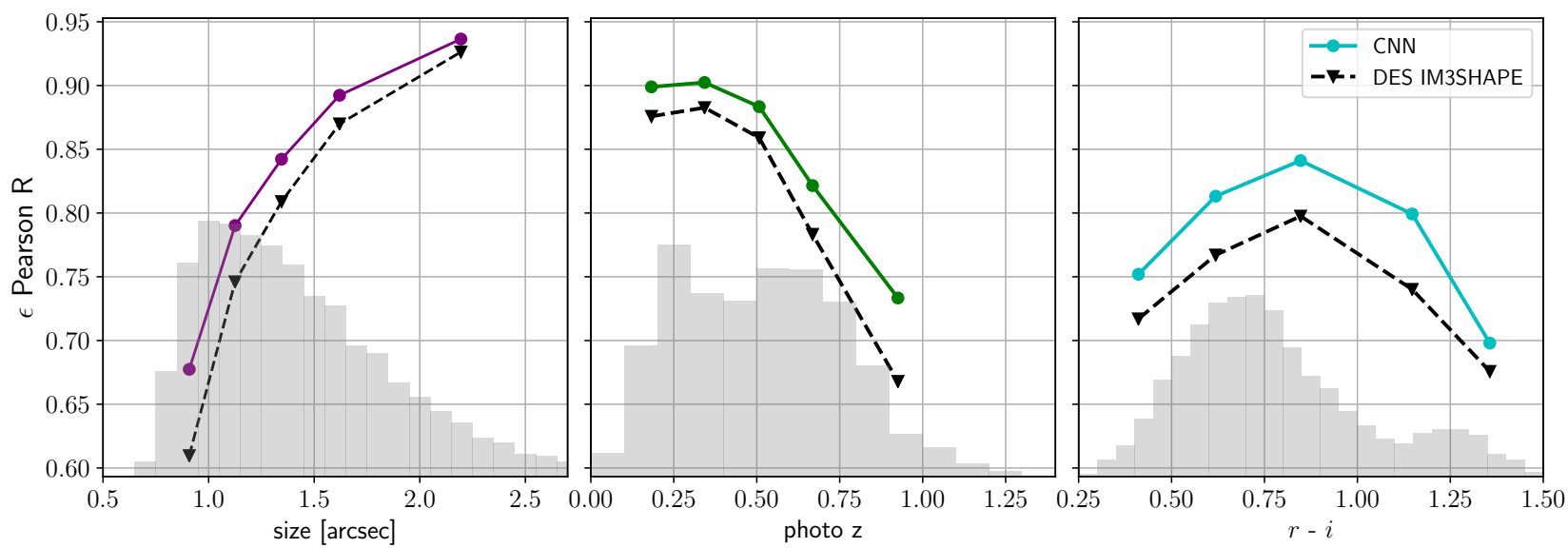

Figure 5. The CNN estimates galaxy shapes more accurately than the DES IM3SHAPE catalogue regardless of galaxy size (left), redshift (center) or galaxy $r-i$ color (right). Only galaxies selected for cosmology in the DES IM3SHAPE catalogue are shown. The $r-i$ color dependence is only shown for galaxies where peak photometric redshift estimate is between $0.6-0.8$.

galaxies. On the other hand, the CNN vastly outperforms IM3SHAPE on the subset of small and faint galaxies which were rightfully excluded from cosmology in the IM3SHAPE catalogue.

We also evaluate performance depending on other measured physical properties of galaxies, such as size, redshift, and color index, to see whether any of these can explain the performance advantage of the CNN. For these tests, we only use the subset selected for cosmology where IM3SHAPE closely matches the performance of the $\mathrm{CNN}$, and galaxies are divided into 5 bins. In the third evaluation with color index, we selected galaxies with peak photometric redshifts between $0.6-0.8$ according to CFHTLenS photo-z estimates (Hildebrandt et al. 2012) to restrict our analysis to galaxy colors resulting from different spectral types. At this redshift, the $4000 A$ break lies on the border of $r$ and $i$ bands, and galaxies clearly show red-blue bimodal distribution. We selected uniform intervals for the color index bin edges in- stead of equal size bins in order to sufficiently cover the less populated mode of red galaxies. We find that the CNN is more accurate than IM3SHAPE regardless of galaxy size, redshift or color index [Fig. 5], however, at large galaxy size, the difference between the methods seems to diminish. The results on distant, faint and small galaxies indicate that the CNN shape estimator could increase the galaxy density of the survey via allowing fewer galaxies to be excluded than with the IM3SHAPE maximum likelihood estimator.

We manually inspected many galaxies where the CNN is more accurate than IM3SHAPE in order to see whether the accuracy difference can be explained by some unusual feature, such as blending or artefacts. We find no such distinguishing features, except for high noise level and the small galaxy size discussed earlier. Finally, we show a few examples of galaxies and estimated ellipticities. We selected two examples where the CNN estimated shapes significantly more accurately than the DES catalog [Fig. 6], one where both 

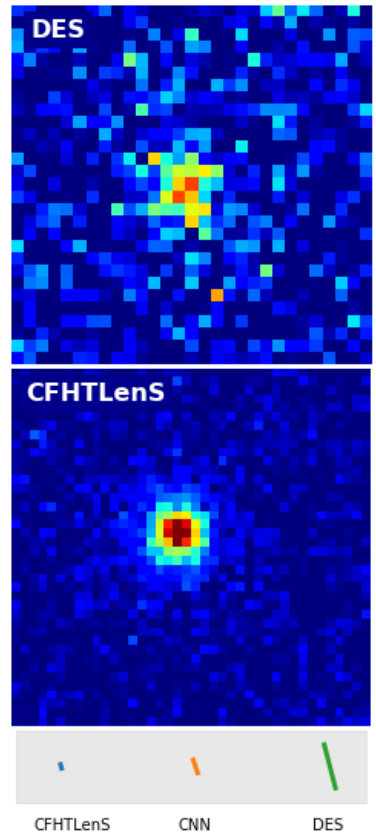
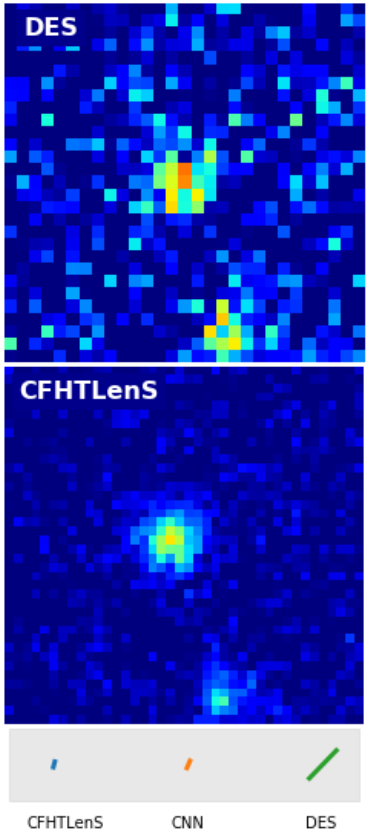
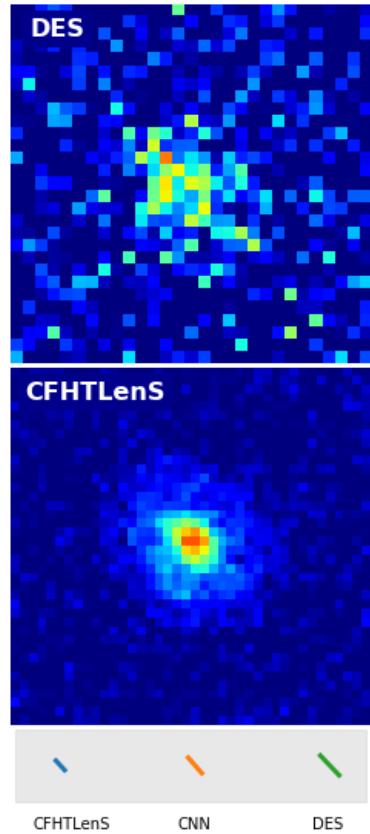
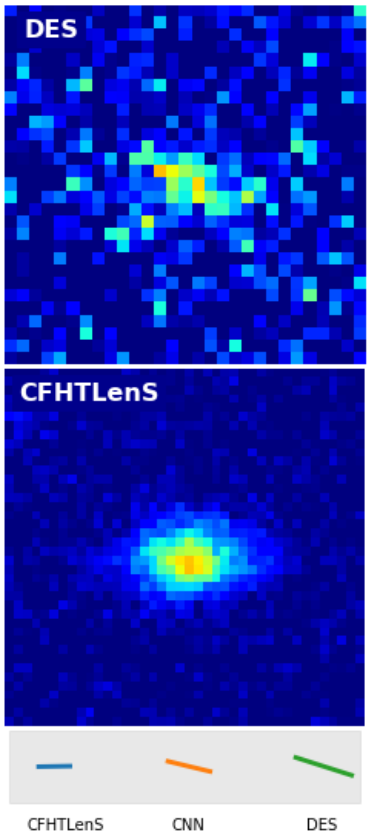

Figure 6. Example galaxy shape measurements and $r$-band images from DES (top) and $i$-band images CFHTLenS (bottom) for 4 example galaxies. The ellipticity estimates are represented with lines at the bottom of the figures. The height of the grey band represents an ellipticity of 0.8 .

were correct, and one where both were wrong. The selected galaxies have high redshifts and large noise and we only selected galaxies where at least 5 different exposures are used when fitting IM3SHAPE. The examples illustrate that galaxy shape estimation is indeed a very hard task for noisy and small examples.

\section{SHAPE ESTIMATION USING MULTI-BAND IMAGES}

Shapes of galaxies are highly correlated in different colours, therefore different band DES images can be combined to achieve more accurate shape measurements of faint galaxies. The most useful colours are riz bands, which are used in the METACALIBRATION catalogue, therefore we also train and evaluate the CNN on images where these 3 bands were stacked in different input channels. All the other details of the neural network and the training protocol is the same as in the one band case. Note that additional input channels have practically no effect of the runtime of the CNN, unlike in the case of forward fitting methods, where there is a linear relationship between the number of images in the joint fit and the runtime of estimation. The first layer in the CNN, which maps from the input channels to 16 filters, is practically negligible compared to the next layer which maps from 16 to 16 filters, regardless of the whether the number of input channels is 1 or 3 . With a much larger number of input channels, the runtime can be dominated by the first layer, and this will inevitably lead to additional computation time, however, with only sub-linear scaling.

We compare the performance of the multi-band CNN to the DES Y1 METACALIBRATION shape catalogue, which used the 'ngmix' engine (Sheldon 2015) to fit a single Gaus- sian galaxy brightness profile (Zuntz et al. 2018), after forward convolution by the PSF which is also approximated with a single Gaussian. The simple representation of both galaxy and PSF profiles with a single Gaussian was reported to be necessary for computational efficiency (Zuntz et al. 2018). Similarly to the IM3SHAPE catalogue, fits were performed on many single epoch observations by maximizing joint likelihood. The ellipticity of the galaxy profiles had a prior strongly centred on zero, which was found to be necessary for stable fits for faint objects. Note that IM3SHAPE does not use such a strong prior on ellipticities which may result in unstable fits for faint galaxies. This prior results in a strong multiplicative bias $(\approx 0.3)$ in raw ellipticity estimates, however, this is entirely corrected with the METACALIBRATION procedure, which we discuss in detail in $\S 6$. Therefore, we use the ensemble corrected shear estimates for the METACALIBRATION catalogue. Note that due to the two additional bands, the METACALIBRATION catalogue is significantly more accurate than the IM3SHAPE catalogue.

It is important to note that in the METACALIBRATION procedure, an extra noise field is added to the images before shape measurement. This noise field has the same r.m.s. as the noise on the original image, effectively reducing the $\mathrm{S} / \mathrm{N}$ of the image by $\sqrt{2}$. Therefore, in order to perform a fair comparison of the CNN and the METACALIBRATION catalogue we evaluate the $\mathrm{CNN}$ on images with an additional noise field with unit variance, called "fixnoise". Predictions on raw images are also shown in order to illustrate the effect of fixnoise. Note that we train the CNN on raw images, and only add extra noise before predictions.

We repeat the same evaluations as in the case of singleband measurements, using only the subset of galaxies selected for cosmology in the DES Y1 METACALIBRATION cat- 

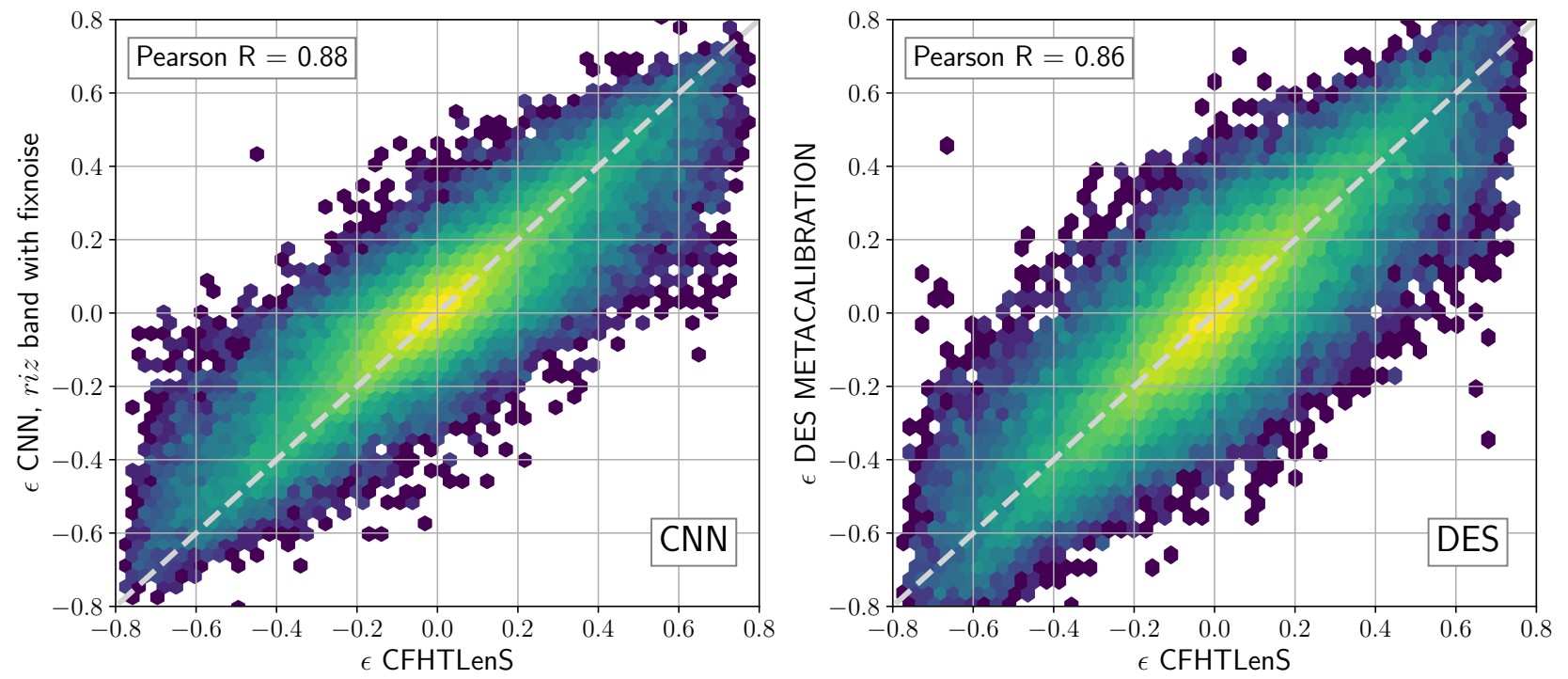

Figure 7. The CNN estimates galaxy shapes with smaller dispersion than the DES METACALIBRATION catalogue. The heatmap shows joint distributions of ground truth CFHTLenS shapes and estimates by the CNN (left) and the DES shape catalogue (right) for galaxies which were selected for cosmology in the DES METACALIBRATION catalogue.

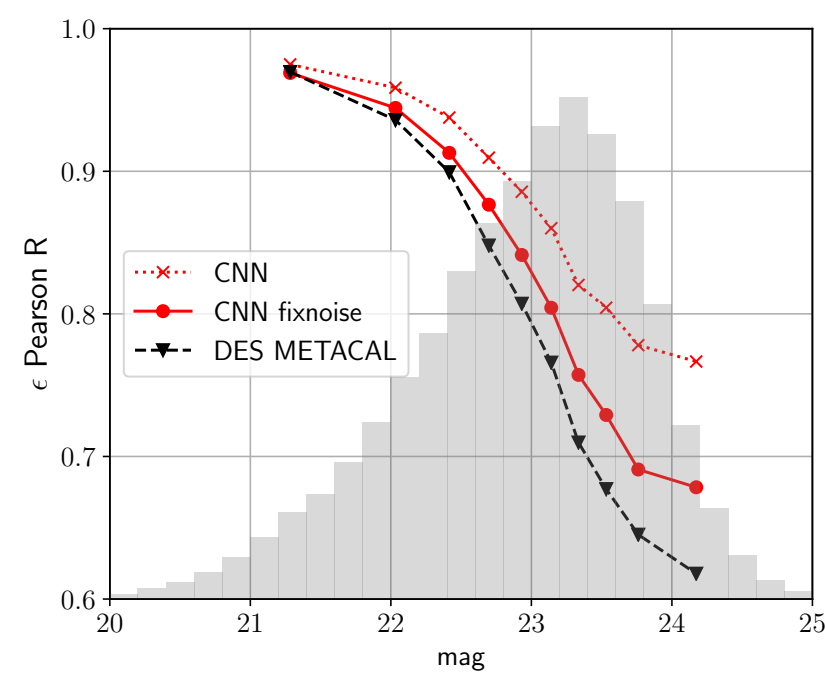

Figure 8. The CNN estimates shapes of faint galaxies more accurately than the DES Y1 METACALIBRATION catalogue. The solid line shows the precision of the CNN shape estimator evaluated on images comparable to DES measurements, with additional artificial noise as in METACALiBration. The dotted line represents CNN shape estimates on original images, it is added to illustrate the effect of additional noise. Only galaxies selected for cosmology in the DES METACALIBRATION catalogue are shown.

alogue. In the METACALIBRATION catalogue the only significant exclusion criteria is $S / N>10$ in order to avoid implicit selection effects from source detection. We find that the CNN estimates shapes of galaxies with smaller dispersion than the DES catalogue when using multi-band images [Fig. 7]. The advantage of the CNN only appears at faint galaxies [Fig. $8]$ and it is independent of the size and the redshift of these galaxies [Fig. 9].

Interestingly, we find that the advantage of the $\mathrm{CNN}$ is increased on blue galaxies [Fig. 9], while it almost disappears for red ones. Blue, star-forming galaxies can have complex, irregular shapes and these are apparently much better characterized by the CNN than a single Gaussian fit. On the other hand, in the case of relaxed, red, elliptical galaxies a Gaussian profile fits the images almost as well as the predictions of the CNN. The CNN is not limited by design to use a small set of simple surface brightness profiles, and our results indicate that it is able to learn a useful prior of possible complex galaxy shapes, which gives it a significant advantage over a single Gaussian profile for distant blue galaxies.

\section{THE EFFECT OF THE SIZE OF THE TRAINING DATASET}

Our training dataset contains approximately $10^{5}$ galaxies, and we investigate the performance depending on the number of galaxies for training the $\mathrm{CNN}$, by randomly excluding galaxies from the training set. We maintain the same number of minibatch iterations regardless of training data size, in order to allow convergence for small training data sizes. For demonstration purposes, we also incorporate $g$ and $Y$ bands into the input, therefore it is a 5 channel image. We find that inclusion of the image bands only very slightly improves performance. The precision of the CNN plateaus around $4 \times 10^{4}$ galaxies [Fig. 10], with very little if any improvement for larger training set sizes. A peculiar flat region appears between $2 \times 10^{3}-10^{4}$ galaxies, but this is probably due to sub-optimal training schedule for some training set sizes. The results indicate that a larger collection of galaxies, such as a simulated dataset, is not expected to significantly improve the dispersion reached here. Future surveys will easily collect the amount of data necessary to train a CNN shape estimator to reach its full potential. 

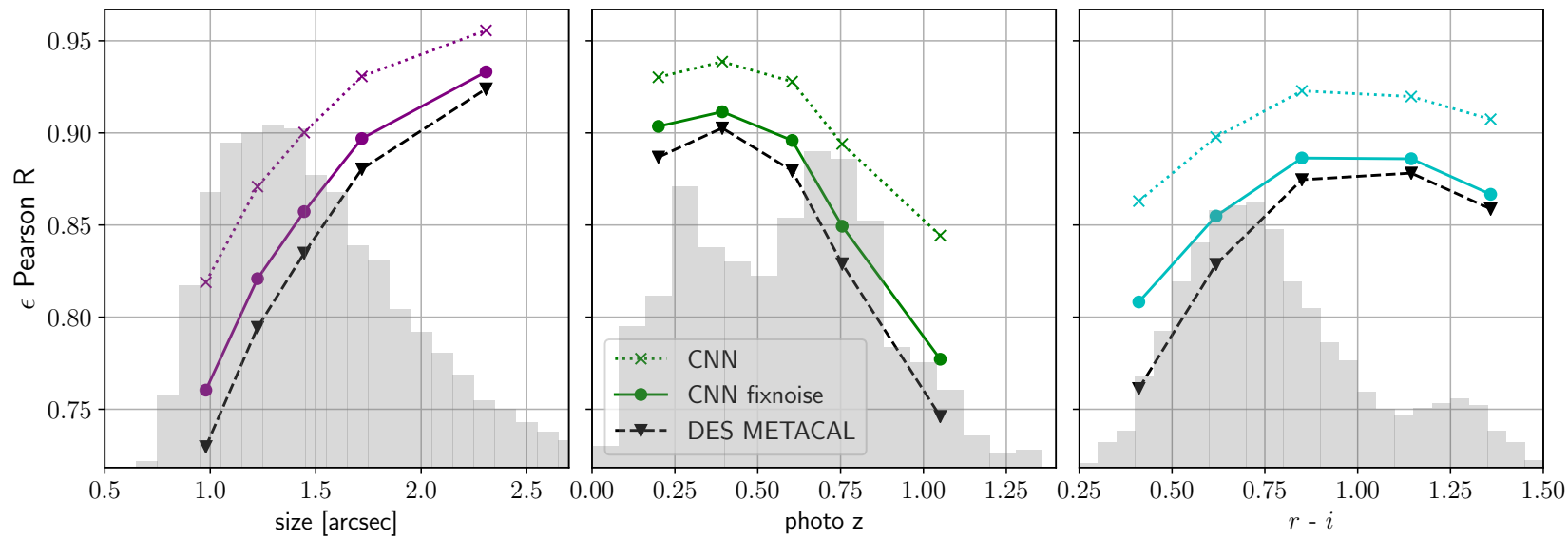

Figure 9. The CNN estimates galaxy shapes more accurately than the DES METACALIBRATION catalogue regardless of galaxy size (left)and redshift (center). The advantage is larger for blue galaxies than red ones (right).

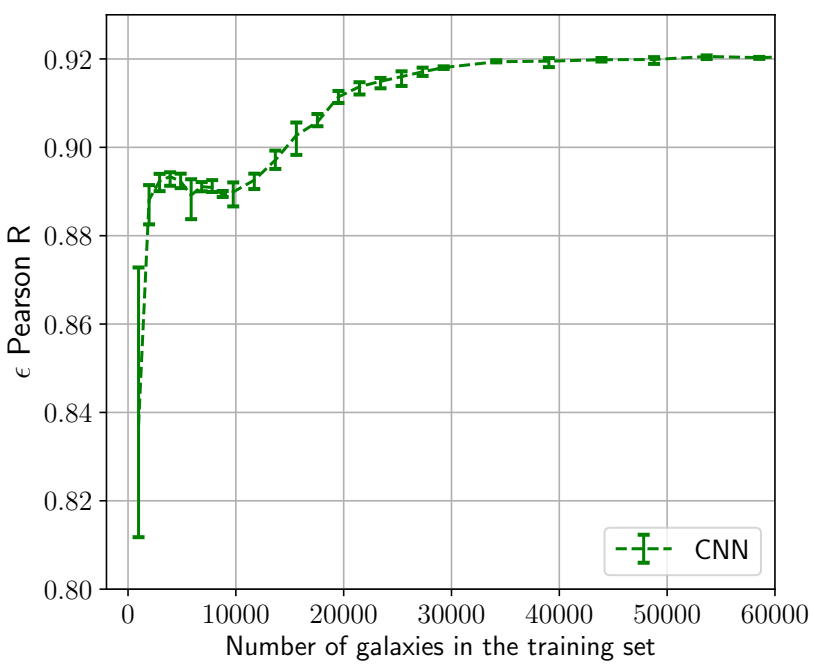

Figure 10. Approximately $4 \times 10^{4}$ images are needed to reach near maximal accuracy with the CNN using grizY band DES images. Errorbars represent minimum and maximum values for 4 runs with different random training subsets.

\section{METACALIBRATION FOR THE CNN}

\subsection{The metacalibration formalism}

METACALIBRATION is a recently developed innovative selfcalibration protocol for weak lensing shear measurements, which calculates necessary corrections using the images themselves instead of deriving them from a large suite of simulated galaxies (Huff \& Mandelbaum 2017; Sheldon \& Huff 2017). METACALIBRATION was successfully implemented to produce one of the shape catalogues of the DES Y1 data release (Zuntz et al. 2018). Without weak lensing, we would measure the unperturbed shape of the galaxy and percent level cosmic shear only very slightly changes the measure- ment. For small enough shears the observed shape can be approximated with a linear relationship to shear.

$\boldsymbol{\epsilon}=\left.\boldsymbol{\epsilon}\right|_{\boldsymbol{g}=0}+\left.\frac{\partial \boldsymbol{\epsilon}}{\partial \boldsymbol{g}}\right|_{\boldsymbol{g}=0} \boldsymbol{g}+O\left(\boldsymbol{g}^{2}\right)$

, where $\boldsymbol{\epsilon}$ is the two component shape estimate and $\boldsymbol{g}$ is the cosmic shear. The partial derivative of shape measurement with respect to shear is called response.

The core idea of METACALIBRATION is that the response can be approximated by artificially shearing and remeasuring the image of the galaxy. Via measuring the shapes of galaxies at different artificial shear values we can calculate the finite derivative of shape measurement with respect to shear. To correctly mimic the effect of cosmic shear, one must first deconvolve the images with the PSF, apply the known artificial shear and then reconvolve image with a circular synthetic PSF. It is practical to calculate a two-sided derivative.

$R_{i, j}=\frac{\epsilon_{i}\left(\Delta g_{j}\right)-\epsilon_{i}\left(-\Delta g_{j}\right)}{2 \Delta g_{j}}$

The mean shape over a set of galaxies is the sum of intrinsic shapes and shapes induced by shear.

$\langle\boldsymbol{\epsilon}\rangle=\left\langle\left.\boldsymbol{\epsilon}\right|_{\boldsymbol{g}=0}\right\rangle+\langle\boldsymbol{R} \boldsymbol{g}\rangle+O\left(\boldsymbol{g}^{2}\right)$

The mean shape of galaxies in the absence of shear has zero expectation value for a well-behaving shape estimator. Assuming constant shear acting on galaxies, the METACALIBRATION shear estimator can be expressed with the mean response and the mean shape over a set of galaxies.

$\langle\boldsymbol{g}\rangle \approx\langle\boldsymbol{R}\rangle^{-1}\langle\boldsymbol{\epsilon}\rangle$

For ellipticity measurements, the response is a diagonal matrix, and the formula reduces to an element-wise division. Note that response is practically equivalent to multiplicative bias, and therefore it is low for faint galaxies, resulting in the implicit down-weighting of shear measured in these galaxies by the shape estimation algorithm itself as $\langle\boldsymbol{\epsilon}\rangle=\langle\boldsymbol{R} \boldsymbol{g}\rangle$. 


\section{2 fixnoise correction}

In the presence of noise, simple METACALIBRATION breaks down. The artificial shear is intended to only shear the galaxy, however, it also shears the deconvolved noise field, therefore it fails to accurately mimic cosmic shear. The additional sheared correlated noise field results in the underestimation of the response (Sheldon \& Huff 2017). In the presence of an asymmetric PSF, another problem also arises. Apart from the light of the galaxy which was smeared by the PSF originally, the white noise field of the image is also deconvolved by the asymmetric PSF, introducing significant PSF leakage.

An additional noise field, with the same dispersion, but opposite shear statistically negates the undesired contribution of the original noise field, this method is called "fixnoise". Practically the new noise field needs to undergo the same operations as the images, rotated by $90 \mathrm{deg}$ and added to the manipulated galaxy image. The final counterfactual image will be symmetric regarding the direction of PSF asymmetry and artificial shear, therefore the shape measurement will not have PSF leakage and the response can be measured correctly.

$$
\widetilde{I}=\widetilde{I_{g a l}}+\widetilde{\eta}\left(\Delta \boldsymbol{g}, \boldsymbol{\epsilon}_{P S F}\right)+\widetilde{\eta_{f i x}}\left(-\Delta \boldsymbol{g},-\boldsymbol{\epsilon}_{P S F}\right)
$$

, where $\widetilde{I}$ is the counterfactual image of the galaxy and $\widetilde{\eta}$ is the distorted noise field. The accuracy of METACALIBRATION with fixnoise correction has been thoroughly verified in simulations by Sheldon \& Huff (2017) and Zuntz et al. (2018).

The shape of the galaxy must also be measured on devonvolved and reconvolved images with additional fixnoise, because we measure the response of this particular shape estimator. The additional noise field reduces the signal to noise ratio of the image by $\sqrt{2}$, reducing the precision of measurements in exchange for principled shear calibration.

\subsection{Simulated galaxies}

In principle METACALIBRATION does not need large simulations which precisely match survey data to produce calibration values, however, it is necessary to test the biases of METACALIBRATION with every new shape estimator, pipeline or survey with a large amount of controlled simulation data resembling the true dataset. We simulate $48 \times 48$ pixel size postage stamp images of $3.5 \times 10^{8}$ bulge+disk composite galaxies using the galsim python package (Rowe et al. 2015), at a scale of 0.263 " per pixel. The disk component has an exponential profile and the bulge has a Sérsic profile with $n=4$. The fraction of flux in the disk component has a uniform distribution between 0 and 1 , and the total flux of the galaxies has a truncated Gaussian distribution, the mean flux was 150, the r.m.s. 125, and minimum flux was 100. We assign uncorrelated ellipticities to the disc and the bulge with Gaussian distributions for the $\epsilon_{1,2}$ components with an r.m.s. of 0.2 and 0.1 respectively. Each galaxy is sheared with a uniform random $g_{1,2}$ value between -0.04 to 0.04. The half light radii of galaxy surface profiles before convolution with the PSF have a truncated Gaussian distribution, with mean 0.75 , r.m.s. 0.25 and minimum 0.2 in units of the half light radius of the PSF. We use a Gaussian

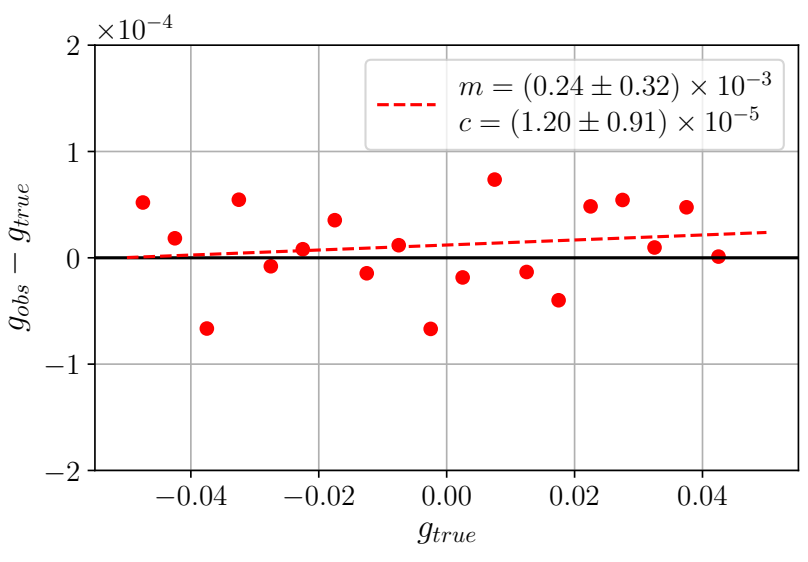

Figure 11. Multiplicative and additive bias of the metacalibrated CNN shear estimator. Measured from 350 million simulated galaxies.

PSF with $F W H M=0.9^{\prime \prime}$, because the convolutional neural network was trained on stacked images with complex PSFs. We assign a uniform random ellipticity to the psf with $\epsilon_{1,2}$ values between -0.025 to 0.025 .

\section{4 metacalibration results}

We implement the METACALIBRATION procedure using CNN shape estimates building on the convolution and shear functions of galsim. We use the CNN described previously, trained only on single band DES images and adopt an artificial shear value $\Delta g=0.001$. The convolution operations of METACALIBRATION dominate the runtime, approximately $40 \mathrm{~ms}$ per galaxy, therefore we use full test-time augmentation by averaging predictions on all 8 flipped and transposed combinations, as it was practically free. The mean response of the CNN shape estimator on the simulation is around 0.7, apparently smaller than in the observational data, showing that the simulation is sufficiently challenging. Multiplicative and additive biases are calculated by fitting a linear function to the error of shear estimates depending on known shear simultaneously for 350 million galaxies. We find that biases of the CNN estimator after METACALIBRATION are the same in the two components, therefore we merge them.

The final multiplicative bias is $m=(0.24 \pm 0.32) \times 10^{-3}$, well below the limit necessary for future large weak lensing surveys. The result is very similar to the one obtained by (Sheldon \& Huff 2017) using their variable shear simulation. The additive bias is also negligible, $c=(1.2 \pm 0.9) \times 10^{-5}$. The results are depicted with the fitted linear relationship and 20 aggregated points [Fig. 11].

In METACALIBRATION, PSF asymmetries are handled by PSF measurement and deconvolution instead of shape measurement algorithms, and it was thoroughly tested on DES Y1 data by Zuntz et al. (2018). They found that PSF measurement errors dominate the potential error introduced by deconvolution. We also characterise the PSF leakage of shape measurement by fitting a linear function to shape estimate errors and PSF ellipticity. We find that PSF leakage is an order of magnitude smaller than the effect of PSF measurement errors in Zuntz et al. (2018) [Fig. 12]. 


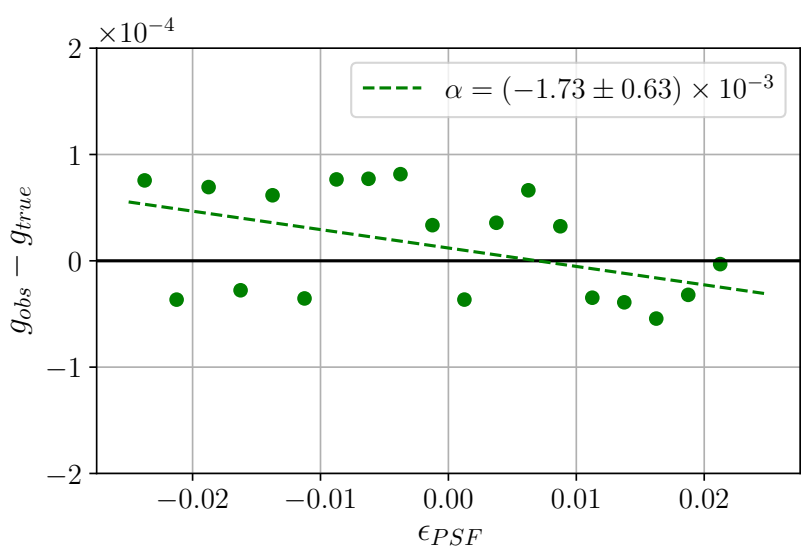

Figure 12. PSF leakage of the metacalibrated CNN shear estimator.

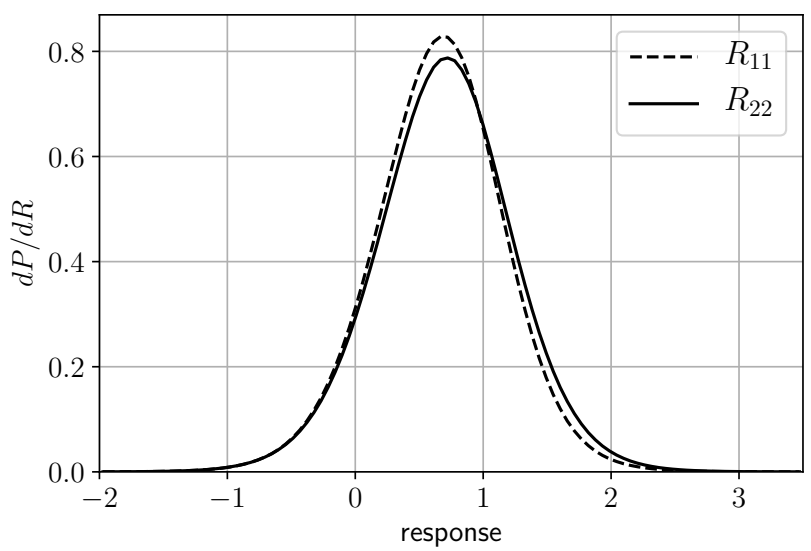

Figure 13. The distribution of the response values of the CNN. Mean response is larger for the diagonal ellipticity component.

Interestingly, the response of the $\mathrm{CNN}$ is different for the two shape components, with a significantly larger response for the second, diagonal component [Fig. 13]. The difference is due to additional shearing operations and the application of fixnoise, because multiplicative biases of the two components are equal on raw simulated images. However, we find no such difference when estimating shapes of these galaxies using adaptive moments (Bernstein \& Jarvis 2002). Note that this peculiarity does not interfere with the METACALIBRATION procedure.

\section{DISCUSSION}

We present a novel setup to create a training and testing dataset from only observational data using a wider but shallower survey to obtain postage stamp images of galaxies, and a narrow but deeper survey to measure the 'ground truth' shapes of galaxies. Such a training and testing setup will also be possible to construct for any ongoing or planned weak lensing survey with dedicated deep fields such as Deep Drilling Field for LSST, Euclid Deep Fields, The Subaru
Deep Field and WFIRST Deep Fields. Space telescope data from Hubble and WJT could also be used to provide "ground truth" shapes.

We propose convolutional neural networks for galaxy shape estimation for weak lensing, and we show that a CNN is able to perform a shape measurement in 0.2 milliseconds, more than $10000 \times$ faster than a maximum likelihood forward fitting method. Model fitting procedures are very resource intensive, which already warrant simplifications in current surveys (Fenech Conti et al. 2017; Zuntz et al. 2018), and it will be a large challenge to tackle large scale, deep surveys in the near future. CNNs offer a super-fast alternative, which could significantly facilitate data analysis for very large weak lensing surveys.

The CNN is able to match the precision of maximum likelihood shape estimates for very bright galaxies and significantly outperforms them on faint galaxies. On the small and faint subset of a galaxies excluded from cosmology in the DES IM3SHAPE catalogue, where the maximum likelihood method struggles to provide reliable shape estimates which can be calibrated, the $\mathrm{CNN}$ is able to measure the shapes of this galaxies with much higher accuracy, potentially preventing the exclusion of some these galaxies for increased galaxy density. The CNN is able to measure galaxy shapes using multi-band input images with no additional overhead compared to the single band case. The precision of these multi-image shape estimates match the DES Y1 METACALIBRATION catalogue at bright galaxies, and it is significantly higher at faint galaxies. The CNN is not limited by simple surface brightness profiles, which enables it to significantly outperform the single Gaussian fit of DES Y1 METACALIBRATION on blue galaxies, while the difference is much smaller on relaxed red galaxies.

Improved precision is worthless if systematics degrade, and we demonstrate that the CNN shape estimator can be nested in the METACALIBRATION process, to yield shear estimates with a sufficiently small multiplicative bias for future large weak lensing surveys $m<10^{-3}$ with no significant PSF leakage. The CNN reaches peak performance around $4 \times 10^{5}$ galaxies, which can be easily collected in future large surveys, and our result indicate that more data might not improve precision much further.

For our proof of concept study we use the easily accessible stacked images from DES DR1, however, weak lensing surveys generally evaluate multi-epoch data with a joint fit over single exposures (Miller et al. 2013; Zuntz et al. 2018) as the interpolation of the PSF creates artefacts in stacked images which are problematic for shear estimation (Miller et al. 2013). A joint prediction using multiple exposures is perfectly possible with CNNs too, as demonstrated by joint prediction on multiple band images. The exploration of CNN shape estimators in extreme cases such as $\approx 1000$ exposures per galaxy in LSST may provide further interesting results.

METACALIBRATION reduces the signal to noise ratio of galaxies and therefore hampers the precision of shear estimates in exchange for robustly eliminating bias. Our current implementation of METACALIBRATION dominates runtime, therefore in order to fully exploit the performance of CNNs this limitation needs to be tackled, possibly with a GPU accelerated METACALIBRATION implementation. The results of Tewes et al. (2019) indicate that a specifically tailored neural network could be able to produce shear estimates with 
small bias and no significant PSF leakage. Tewes et al. (2019) provide properties of the galaxy and the PSF as inputs to a densely connected neural network, and a CNN could also have access to all the necessary information and it could also be specifically trained to natively produce shear estimates with small bias and PSF leakage. Such a shape estimator could fully realise the potential of CNNs both in terms of precision and speed.

Further tests with CNN shape estimators in simulations could improve our understanding of factors which contribute to the performance advantage of the CNN compared to a maximum likelihood model fitting approach. The extraction of meaningful and interpretable knowledge from the inspection of a CNN could also improve our understanding of the problem itself (Ribli et al. 2019b). Finally, in our proposed scheme, galaxy shape estimation with CNNs cannot completely replace model fitting approaches, as the training procedure relies on high-quality shape measurements from a deeper survey which must be performed with conventional methods.

\section{ACKNOWLEDGEMENTS}

We thank the anonymous referee, for the report which helped us significantly improve the manuscript, and we thank Joe Zuntz and Erin Sheldon for useful discussions and comments. This work was partially supported by the National Research, Development and Innovation Office of Hungary via grant OTKA NN 129148 and the National Quantum Technologies Program.

\section{REFERENCES}

Abbott T. M. C., et al., 2018, ApJS, 239, 18

Amendola L., et al., 2018, Living Reviews in Relativity, 21, 2

Bacon D. J., Refregier A. R., Ellis R. S., 2000, MNRAS, 318, 625

Bernstein G. M., Jarvis M., 2002, AJ, 123, 583

Bertin E., Arnouts S., 1996, A\&AS, 117, 393

Bridle S., et al., 2009, Annals of Applied Statistics, 3, 6

Bridle S., et al., 2010, MNRAS, 405, 2044

Chang C., et al., 2013, MNRAS, 434, 2121

Cohen T., Welling M., 2016, in International conference on machine learning. pp 2990-2999

Diehl H. T., et al., 2016, in Observatory Operations: Strategies, Processes, and Systems VI. p. 99101D, doi:10.1117/12.2233157

Dieleman S., Willett K. W., Dambre J., 2015, MNRAS, 450, 1441

Dobos L., Budavári T., Li N., Szalay A. S., Csabai I., 2012. (arXiv: 1206.5021)

Domínguez Sánchez H., Huertas-Company M., Bernardi M., Tuccillo D., Fischer J. L., 2018, MNRAS, 476, 3661

Erben T., et al., 2013, MNRAS, 433, 2545

Fenech Conti I., Herbonnet R., Hoekstra H., Merten J., Miller L., Viola M., 2017, MNRAS, 467, 1627

He K., Zhang X., Ren S., Sun J., 2015, in Proceedings of the IEEE international conference on computer vision. pp 1026-1034

He K., Zhang X., Ren S., Sun J., 2016, in Proceedings of the IEEE conference on computer vision and pattern recognition. pp $770-778$

Herbel J., Kacprzak T., Amara A., Refregier A., Lucchi A., 2018, J. Cosmology Astropart. Phys., 7, 054

Heymans C., et al., 2006, MNRAS, 368, 1323

Heymans C., et al., 2012, MNRAS, 427, 146
Heymans C., et al., 2013, MNRAS, 432, 2433

Hildebrandt H., et al., 2012, MNRAS, 421, 2355

Hildebrandt H., et al., 2017, MNRAS, 465, 1454

Hirata C., Seljak U., 2003, MNRAS, 343, 459

Huff E., Mandelbaum R., 2017, arXiv e-prints,

Ioffe S., Szegedy C., 2015, arXiv preprint arXiv:1502.03167

Jarvis M., Jain B., 2008, J. Cosmology Astropart. Phys., 1, 003

Jarvis M., et al., 2016, MNRAS, 460, 2245

Kacprzak T., Zuntz J., Rowe B., Bridle S., Refregier A., Amara A., Voigt L., Hirsch M., 2012, MNRAS, 427, 2711

Kacprzak T., Bridle S., Rowe B., Voigt L., Zuntz J., Hirsch M., MacCrann N., 2014, MNRAS, 441, 2528

Kaiser N., 2000, ApJ, 537, 555

Kaiser N., Squires G., Broadhurst T., 1995, ApJ, 449, 460

Kaiser N., Wilson G., Luppino G. A., 2000, arXiv Astrophysics e-prints,

Kilbinger M., 2015, Reports on Progress in Physics, 78, 086901

Kitching T. D., Miller L., Heymans C. E., van Waerbeke L., Heavens A. F., 2008, MNRAS, 390, 149

Kitching T., et al., 2010a, arXiv e-prints,

Kitching T., et al., 2010b, Gravitational Lensing Accuracy Testing 2010 (GREAT10) Challenge Handbook. (arXiv: 1009.0779)

Kondor R., Lin Z., Trivedi S., 2018, in Advances in Neural Information Processing Systems. pp 10138-10147

Krizhevsky A., Sutskever I., Hinton G. E., 2012, in Advances in neural information processing systems. pp 1097-1105

Kuijken K., 1999, A\&A, 352, 355

Kuijken K., et al., 2015, MNRAS, 454, 3500

Lintott C. J., et al., 2008, MNRAS, 389, 1179

Mandelbaum R., et al., 2015, MNRAS, 450, 2963

Mandelbaum R., et al., 2018, Monthly Notices of the Royal Astronomical Society, 481, 3170

Massey R., et al., 2007, MNRAS, 376, 13

Melchior P., Viola M., 2012, MNRAS, 424, 2757

Melchior P., Böhnert A., Lombardi M., Bartelmann M., 2010, A\&A, 510, A75

Miller L., Kitching T. D., Heymans C., Heavens A. F., van Waerbeke L., 2007, MNRAS, 382, 315

Miller L., et al., 2013, MNRAS, 429, 2858

Morganson E., et al., 2018, PASP, 130, 074501

Pujol A., Kilbinger M., Sureau F., Bobin J., 2019, A\&A, 621, A2

Redmon J., Farhadi A., 2017, arXiv preprint

Refregier A., Kacprzak T., Amara A., Bridle S., Rowe B., 2012, MNRAS, 425, 1951

Ribli D., Ármin Pataki B., Zorrilla Matilla J. M., Hsu D., Haiman Z., Csabai I., 2019a, arXiv e-prints,

Ribli D., Pataki B. Á., Csabai I., 2019b, Nature Astronomy, 3, 93

Rowe B., et al., 2014, arXiv:1407.7676 [astro-ph]

Rowe B. T. P., et al., 2015, Astronomy and Computing, 10, 121

Schrabback T., et al., 2010, A\&A, 516, A63

Sheldon E., 2015, Astrophysics Source Code Library

Sheldon E. S., Huff E. M., 2017, ApJ, 841, 24

Simet M., Mandelbaum R., Rowe B., Great3 Collaboration 2014, $223,255.17$

Simonyan K., Zisserman A., 2014, arXiv preprint arXiv:1409.1556

Springer O. M., Ofek E. O., Weiss Y., Merten J., 2018, arXiv e-prints,

Szegedy C., et al., 2015.

Takada M., 2010, in AIP Conference Proceedings. pp 120-127

Tewes M., Kuntzer T., Nakajima R., Courbin F., Hildebrandt H., Schrabback T., 2019, A\&A, 621, A36

Tuccillo D., Huertas-Company M., Decencière E., Velasco-Forero S., 2017, ] 10.1017/S1743921317000552, 325, 191

Van Waerbeke L., et al., 2000, A\&A, 358, 30

Voigt L. M., Bridle S. L., 2010, MNRAS, 404, 458

Wang H., et al., 2019, Nat. Methods, 16, 103 
Wittman D. M., Tyson J. A., Kirkman D., Dell'Antonio I., Bernstein G., 2000, Nature, 405, 143

Xu L., Ren J. S., Liu C., Jia J., 2014, in Advances in Neural Information Processing Systems. pp 1790-1798

Zuntz J., Kacprzak T., Voigt L., Hirsch M., Rowe B., Bridle S., 2013, MNRAS, 434, 1604

Zuntz J., et al., 2018, MNRAS, 481, 1149

This paper has been typeset from a $\mathrm{T}_{\mathrm{E}} \mathrm{X} / \mathrm{LAT}_{\mathrm{E}} \mathrm{X}$ file prepared by the author. 